\title{
Extending Monte Carlo Simulations of Electron Microscopy Images and Hyperspectral Images in a User-Friendly Framework
}

\author{
Samantha Rudinsky ${ }^{1}$, Yu Yuan ${ }^{1}$, Raynald Gauvin ${ }^{1}$, Nicolas Piché $^{2}$, Mike Marsh $^{3}$ \\ 1. Department of Materials Engineering, McGill University. Montreal, Canada. \\ 2. Object Research Systems. Montreal, Canada. \\ 3. Object Research Systems. Denver, USA.
}

Characterization by electron microscopy plays a crucial role in research across a variety of materials research applications because it can be used to discern the physical and chemical properties of the studied materials. However, in order to make large scale assessments of new products and structures, such techniques need to be automated and optimized so that large amounts of data may be processed efficiently. Optimization can sometimes be done empirically by varying imaging conditions on an available microscope, but when those imaging conditions can be varied through simulation, it is easier to test more systematically a wider variety of conditions. MC X-ray [1] is such a simulation tool which can compute the complete backscattered electron (BSE) images and x-ray spectra from the simulation of electron scattering in solids of various geometries. Until recently, the flexibility of simulated sample domains was constrained by limits in the MC X-ray user interface. We present here the integration of the simulation technology of MC X-ray in a feature-rich graphical image processing and image visualization platform, enabling greater user flexibility and the capability to use previously collected images as the initial spatial domain of simulation.

MC X-ray provides users the capability to simulate electron scattering and concomitant x-ray emission for spatial systems of different materials. Users can define sample geometries with planes, spheres, and cylinders, but no graphical interactive tools are provided for constructing and editing complex geometries. Output images can be exported for subsequent analysis in feature-rich image processing software, but such image processing is not integrated, therefore making iterative experiments tedious or impossible.

We present here the integration of the simulator behind MC X-ray into Dragonfly, a software platform for image analysis that is free for non-commercial use. Dragonfly has previously been shown useful for the inspection and analysis of hyperspectral images (HSI) and other high-dimensional signals [2]. Dragonfly has native support for a variety of microscopy images of varying bit-depth and spatial resolution, and it has data structures for encoding image labels that denote the spatial arrangement of the constituent materials in an observed image. These data structures make up the Dragonfly image segmentation framework, and they are very flexible for image visualization, image quantification, and image editing. With this integration, it is now possible to import any empirical image (2D or 3D), use 
existing tools to label the materials, and then simulate the images and spectra that the same sample would produce under a variety of imaging conditions. This is highly extensible as users could assign any arbitrary chemical information to the labeled image.

The integration here has made it possible to investigate both real and fabricated material samples (of any spatial complexity) to predict the imaging and spectral contrast that can be expected under different conditions. This makes it now possible to tune imaging conditions and guide subsequent imaging and spectral experiments, as well as identify practical limits for contrast in certain material systems at a prescribed resolution. The ability to fabricate HSI with real-world spatial geometries and material constituents also opens the door to creating libraries of training data that can inform machine learning solutions which try to segment or label images of unknown composition.

Our results here provide extensive user-friendliness to extend the applications and speed of parameter tuning for simulation. Because we have developed this as an extension and deposited it in Infinite Toolbox, the online repository for Dragonfly extensions, it is free for anyone to download to use or modify.

\section{References:}

[1] R. Gauvin, et al., Microscoscopy and Microanalysis 15 (Suppl 2) (2009), p. 488-489.

[2] N. Piché, et al., Microscoscopy and Microanalysis 24 (Suppl 1) (2018), p. 560-561. 